\title{
On the Dirac Approach to Constrained Dissipative Dynamics
}

\author{
Sonnet Q. H. Nguyen ${ }^{b}$, Eukasz A. Turski ${ }^{b} \sharp$ \\ b Center for Theoretical Physics, Polish Academy of Sciences, Al. \\ Lotników 32/46, 02-668 Warsaw, Poland. \\ * Institute of Mathematics, Polish Academy of Sciences, Śniadeckich 8, \\ P.O. box 137, 00-950 Warsaw, Poland. \\ $\sharp$ College of Sciences, Al. Lotników 32/46, 02-668 Warsaw, Poland. \\ E-mails: sonnet@cft.edu.pl and laturski@cft.edu.pl
}




\begin{abstract}
In this paper we propose a novel algebraic and geometric description for the dissipative dynamics. Our formulation bears some similarity with the Poisson structure for non-dissipative systems. We develop a canonical description for constrained dissipative systems through an extension of the Dirac brackets concept, and we present a new formula for calculating Dirac brackets. This formula is particularly useful in description of dynamical systems with many second-class constraints. After presenting necessary formal background we illustrate our method on several examples taken from particle dynamics, continuum media physics and wave mechanics.
\end{abstract}




\section{Introduction}

The first systematic attempt to provide mathematically consistent quantization procedure for constrained systems was given by P.M. Dirac [1], who derived a formal "replacement" for the canonical Poisson brackets which plays today a fundamental role in the canonical formalism for constrained Hamiltonian systems on both classical and quantum levels. In spite of the considerable attention paid to that formula in the mathematical literature [2, 3, 江, and several attempts to use the Dirac brackets in the quantization of gauge invariant systems, eg. [5, 6] etc., until recently there were few attempts to actually use this formalizm in more conventional applications. We have recently provided few examples of these applications in classical and continuum mechanics [7].

The canonical formalizm applies to the conservative systems. These systems form a relatively small sub-class of interesting physical systems since most of the others describing phenomena at some effective rather than fundamental level are dissipative. In the past several attempts were done to describe dissipative classical mechanics in a fashion similar to its canonical description. One of these attempts, so called metriplectic approach [8, 9] was advocated as a natural extension of the mixed canonical-dissipative dynamic proposed by Enz 10]. In this paper we extend further the concept of metriplectic dynamics to what we shall call semimetric-Poissonian dynam$i c s$, which is nothing but a natural combination of semimetric dynamics (a dissipative part) and Poissonian dynamics (a conservative part). We propose a canonical description for constrained dissipative systems through an extension of the concept of Dirac brackets [1] developed originally for conservative constrained Hamiltonian dynamics, to the non-Hamiltonian, namely metric and mixed metriplectic, constrained dynamics. It turns out that this generalized unified formula for the Dirac brackets is very useful in the description and analysis of a wider class of dynamical systems. To proceed with our approach we develop a new formula for calculating Dirac brackets which is particularly effective in finding equations of motion and constants of motions for systems with many constraints.

In order to make this paper self-contained we include in Section II a short "primer" to the Poisson geometry, dynamics and the Dirac brackets.

The rest of the paper is organized into four sections. In Section III we discuss semimetric dynamics and some elementary features of the related

mathematical structures like semimetric algebras and SJ-identity the late should be regarded as dynamical symmetric version of the Jacobi identity. 
We develop symmetric concepts in the analogy with those in the Poisson category in Appendix.

In Section IV we discuss semimetric dynamics subject to some constraints. We derive a symmetric analogue of the Dirac brackets and provide its geometric interpretation as induced metric on some submanifold of a Riemannian manifold. We also present a new effective algorithm for calculation of the Dirac brackets in both symmetric and antisymmetric cases. Few examples, for finite and infinite dimensional cases, are also discussed in some detail.

In the section $\mathrm{V}$ we discuss semimetric-Poissonian dynamics which is a combination of semimetric dynamics, discussed in the section III, and Poissonian dynamics mentioned in the section II. This section also includes the extended Dirac approach to constrained semimetric-Poissonian which is a combination of the Dirac approach to constrained semimetric dynamics discussed in the section IV and the usual to constrained Poissonian dynamics.

In Section VI we discuss interesting physical examples: the dissipative formulation of the Schrödinger equation due to Gisin [11], the LandauLifshitz-Gilbert equations for damped spins [8, 12], dynamics of damped rigid body - including among other the Morrison equation of damped rigid body [9]. We also discuss a novel description of the incompressible viscous fluid.

Some important but not crucial mathematical aspects of the extended canonical formalism for constrained metric and mixed semimetric-Poissonian systems will be given in the forthcoming publication [13]. Computational aspects of the Dirac brackets - symmetric, antisymmetric or mixed - will be publish shortly [14].

\section{Poisson geometry, dynamics and Dirac brackets}

From the algebraic point of view the Poisson algebra is a linear space $\mathcal{F}$ equipped with two structures:

i) the commutative algebra structure with the (associate) multiplication $\mathcal{F} \times \mathcal{F} \rightarrow \mathcal{F}$, the product of two elements $f, g$ is denoted simply by $f g$,

ii) the Lie algebra structure with the Lie bracket $\{\cdot, \cdot\}: \mathcal{F} \times \mathcal{F} \rightarrow \mathcal{F}$, the product of two elements $f, g$ is denoted by $\{f, g\}$,

related to each other by the Leibniz rule:

$$
\{f g, h\}=f\{g, h\}+\{f, h\} g .
$$


The Lie bracket of a Poisson algebra is called the Poisson bracket.

The Poisson manifold is a smooth manifold $M$ for which the commutative algebra of smooth functions on $M, C^{\infty}(M)$, is equipped with the Poisson bracket. The Poisson bracket $\{\cdot, \cdot\}$ acts on each function as a derivation, thus there exists a contravariant $(2,0)$-tensor $\Pi$ such that $\{f, g\}=\Pi(d f, d g)$ for every functions $f, g$. In the local coordinates $\left(z^{k}\right)$

$$
\{f, g\}(z)=\sum_{i, j}^{m} \Pi^{i j}(z) \partial_{i} f \partial_{j} g, \partial_{k} \equiv \frac{\partial}{\partial z^{k}} .
$$

The tensor $\Pi$ which defines a Poisson bracket is called a Poisson tensor. The antisymmetry of Poisson bracket implies that tensor $\Pi$ must be antisymmetric, so $\Pi^{i j}=-\Pi^{j i}$. The Jacobi identity requires that

$$
\sum_{l=1}^{N} \Pi^{l i} \partial_{l} \Pi^{j k}+\Pi^{l j} \partial_{l} \Pi^{k i}+\Pi^{l k} \partial_{l} \Pi^{i j}=0 .
$$

A Hamiltonian vector field generated by a function $h$ is a vector field defined by $X_{h}(f)=\{f, h\}$ for $\forall f$. All flows generated by Hamiltonian vector fieldsHamiltonian flows, preserve the Poisson structure.

Poisson dynamics or generalized Hamiltonian dynamics is a dynamics generated by some Hamiltonian vector field, for which the Hamiltonian function plays physically specific role.

Let $T M$ and $T^{*} M$ denotes the tangent, and cotangent bundle of the manifold $M$. The Poisson tensor $\Pi$ induces a bundle map $\Pi^{\sharp}: T^{*} M \rightarrow T M$ which is defined by $\Pi^{\sharp}(d f):=X_{f}$ for all functions $f$. The rank of a Poisson structure at point $z$ is defined to be the rank of $\Pi_{z}^{\sharp}: T_{z}^{*} M \rightarrow T_{z} M$, which is equal to the rank of matrix $\Pi^{i j}(z)$ in the local coordinates $\left(z^{k}\right)$. A Poisson structure with constant rank equal to the dimension of the manifold $M$ is called symplectic or nondegenerate. In this case the "inverse map" of $\Pi$, denoted by $\omega$, is a symplectic 2-form and $\omega\left(X_{f}, X_{g}\right)=\{f, g\}=\Pi(d f, d g)$. The Darboux theorem states that for every symplectic structure there exists, locally, a canonical coordinates system $\left(x_{1}, \ldots, x_{k}, p_{1}, \ldots, p_{k}\right)$ such that $\Pi=\frac{\partial}{\partial \mathbf{x}} \wedge \frac{\partial}{\partial \mathbf{p}},(\omega=d \mathbf{x} \wedge d \mathbf{p})$, or equivalently, $\left\{x_{i}, x_{j}\right\}=\left\{p_{i}, p_{j}\right\}=0$, $\left\{x_{i}, p_{j}\right\}=\delta_{i j}$.

The invariance of the Poisson structure under Hamiltonian flows implies the constancy the tensor $\Pi$ rank along the orbits of such flows. The orbit of each point of $M$ under the action of all Hamiltonian flows forms a symplectic manifold called a symplectic leave. Since $M$ is a union of such orbits 
every Poisson manifold is a smooth union of disjoint connected symplectic manifold (symplectic leaves) of various ranks.

The splitting theorem [15] for Poisson manifolds, states that locally every Poisson manifold is the product of a symplectic manifold and a Poisson manifold with zero rank. In the other words, locally in the neighborhood of the point $\mathbf{z}_{0}$ there always exist canonical coordinates system:

$\left(x_{1}, \ldots, x_{k}, p_{1}, \ldots, p_{k}, z_{2 k+1}, \ldots, z_{n}\right)$ such that $\left\{x_{i}, x_{j}\right\}=\left\{p_{i}, p_{j}\right\}=0,\left\{x_{i}, p_{j}\right\}=$ $\delta_{i j},\left\{x_{i}, z_{l}\right\}=\left\{p_{j}, z_{l}\right\}=0,\left\{z_{r}, z_{s}\right\}=A_{r s}$ and $A_{r s}\left(\mathbf{z}_{0}\right)=0$.

The map $\varphi: M_{1} \rightarrow M_{2}$ between two Poisson manifolds is called Poisson mapping iff $\{f \circ \varphi, g \circ \varphi\}_{1}=\{f, g\}_{2} \circ \varphi$. The Poisson mapping is a natural generalization of the well-known from classical mechanics notion of the canonical transformation.

In the usual formulation of the classical mechanics the constrained dynamics can be visualized geometrically as the dynamics on some submanifold of the system phase space. Similarly, the constrained Poisson dynamics can be represented as such on some submanifold of the Poisson manifold. However, it is not always possible to define induced Poisson structure on a submanifold and therefore we have no obvious way how to generalize constrained Poisson dynamics. If the Poisson structure is non-degenerate (symplectic case) then on each submanifold there exists an induced two form which becomes symplectic if it is non-degenerate. Further for an arbitrary submanifold $N$ of a symplectic manifold $(M, \omega)$ there always exists a maximal submanifold $N^{\prime} \subset N$ such $\omega_{\mid N^{\prime}}$ is non-degenerate, then $N^{\prime}$ has an induced symplectic hence Poisson structure.

Dirac [1] has proposed an algebraic procedure to deal with constrained dynamics. First consider any finite set of (linearly independent) constraints $\mathcal{A}=\left\{a_{1}, \ldots, a_{k}\right\} \subset \mathcal{F} \equiv C^{\infty}(M)$ and define weakly-vanishing (or weaklyzero) elements as linear combinations of constraints with arbitrary functions coefficients, i.e. $\mathcal{I}=\left\{\sum_{i} f_{i} a_{i}\right.$, where $\left.f_{i} \in \mathcal{F}, a_{i} \in \mathcal{A}\right\}$.

Element $f \in \mathcal{F}$ is called first-class with respect to the set of constraints $\mathcal{A}$ iff it has weekly-zero bracket with all constraints, i.e. $\forall a \in \mathcal{A}:\{f, a\} \in \mathcal{I}$. Otherwise, it is called second-class. The set of all first-class elements denoted by $\mathcal{F}_{1}(\mathcal{A})$, forms a linear subspace of $\mathcal{F}$ and the set of all second-class elements, denoted by $\mathcal{F}_{2}(\mathcal{A})$.

This classification divides the set of constraints $\mathcal{A}$ into two subsets: first-class constraints $\mathcal{A}_{1}=\mathcal{A} \cap \mathcal{F}_{1}(\mathcal{A})$ and second-class constraints $\mathcal{A}_{2}=\mathcal{A} \cap \mathcal{F}_{2}(\mathcal{A})$. The number of second-class constraints must be even $\mathcal{A}_{2}=\left\{\Theta_{1}, \ldots, \Theta_{2 s}\right\}$. Dirac has proven the Gramm matrix of second-class constraints, $\left[\left\{\Theta_{i}, \Theta_{j}\right\}\right]=$ $W$, is weakly non-degenerate. This allowed him to define new antisymmetric 
Leibniz bracket, known as the Dirac bracket:

$$
\{f, g\}_{D}=\{f, g\}-\sum_{i, j=1}^{2 s}\left\{f, \Theta_{i}\right\} C_{i j}\left\{\Theta_{j}, g\right\}
$$

where $C=\left[C_{i j}\right]=W^{-1}$ is an inverse matrix of $W$. Using (2.4) one can check that the Dirac brackets posses all the required properties of the Poisson brackets. The (algebraic) proof of the Jacobi identity is difficult. One can easily check that all the second-class constraints are Casimirs with respect to the Dirac bracket, i.e. $\left\{\Theta_{k}, f\right\}_{D}=0$ for all $f$.

\section{Semimetric manifolds and semimetric dynami- cal systems}

In this section we introduce a concept of semimetric algebras and semimetric manifolds which play a similar role in the description of dissipative systems as the Poisson algebras and the Poisson manifolds in the description of conservative dynamics. In the last two sections of this work we will show that the semimetric structure together with the Poisson structure are sufficient for "canonical" description of a wide class of dissipative dynamical systems.

Let $X$ be non-empty set. A semimetric bracket on the linear space of real functions defined on $X$, namely $\mathcal{F}=F u n(X)$, is a bilinear operation $\prec \cdot, \succ: \mathcal{F} \times \mathcal{F} \rightarrow \mathcal{F}$ which satisfies the following requirements:

i) It is symmetric: $\forall f, g \in \mathcal{F}: \prec f, g \succ=\prec g, f \succ$.

ii) It satisfies the Leibniz rule: $\forall f, g, h \in \mathcal{F}: \quad \prec f g, h \succ=\prec f, h \succ$ $g+f \prec g, h \succ$.

iii) It is non-negative definite: $\forall f \in \mathcal{F}: \prec f, f \succ \geq 0$, i.e. the function $\prec f, f \succ$ is non-negative definite function, $\forall x \in X: \prec f, f \succ(x) \geq 0$.

Note that if a bilinear operation on $\mathcal{F}$ satisfies conditions $\mathbf{i}$ ), ii) it is called pseudo-metric bracket or symmetric Leibniz bracket. A symmetric Leibniz bracket which satisfies the condition

iii*) Positive definite: $\forall f \in \mathcal{F}: \prec f, f \succ \geq 0$ and $\prec f, f \succ=0$ iff $f=$ Const (at least locally), 
is called metric bracket.

Symmetric Leibniz algebra is a linear space $\mathcal{F}$ equipped with two structures: commutative algebra structure with the (associate, commutative) multiplication and symmetric (non-associate, non-commutative) structure and these two structures are related by the Leibniz rule. Semimetric algebra is a symmetric Leibniz algebra whose bracket is semimetric.

As we shall see the semimetric algebra can be used to describe the (wide class) of the dissipative classical systems akin to the description of the nondissipative dynamics by means of the Poisson algebra.

From now we assume that $X$ is a smooth finite dimensional manifold, namely Phase space, and $\mathcal{F}=C^{\infty}(X)$ is a space of all smooth functions on $X$.

There is a correspondence one to one between the symmetric Leibniz brackets on the space of functions and the symmetric tensors on the manifold $X$ : $\prec f, g \succ=G(d f, d g)$ where $G$ is a contravariant tensor field of the type $(2,0)$ on $X$. In the local coordinates $\left(z^{k}\right)$, each symmetric tensor is of the form $G(\mathbf{z})=\sum_{i, j} G^{i j}(\mathbf{z}) \frac{\partial}{\partial z_{i}} \otimes \frac{\partial}{\partial z_{j}}$, where $G^{i j}=G^{j i}$, hence each symmetric Leibniz bracket locally must be of the form:

$$
\prec f, g \succ(z)=\sum_{i, j=1}^{N} G^{i j}(z) \frac{\partial f}{\partial z^{i}} \frac{\partial g}{\partial z^{j}}, f, g \in C^{\infty}(X) .
$$

A symmetric bracket becomes semimetric bracket iff it is non-negative, i.e. the matrix $\left[G^{i j}\right]$ is non-negative definite.

A semimetric bracket is called a metric bracket if $G$ is positive definite (nonnegative and non-degenerate), i.e. with constant maximal rank, $\operatorname{rank} G=$ $\operatorname{dim} X$. In the general the tensor $G$ may have a nonconstant rank which depends on points.

Definition 3.1 Semimetric manifold is a smooth manifold $M$ for which the commutative algebra of smooth functions on $M$ is a semimetric algebra, i.e. it is equipped with a semimetric bracket. Geometrically, semimetric manifold can be viewed as a pair $(M, G)$ where $G$ is semimetric tensor (or cometric tensor), i.e. symmetric, non-negative definite: $G(d f, d f) \geq 0$ for every function $f$, contravariant $(2,0)$-tensor field.

Example 3.1 Tensor $G(\mathbf{z})=\sum_{i=1}^{k} \frac{\partial}{\partial z_{i}} \otimes \frac{\partial}{\partial z_{i}}-\sum_{j=k+1}^{n} \frac{\partial}{\partial z_{j}} \otimes \frac{\partial}{\partial z_{j}}$ is symmetric and non-degenerate, but it is not non-negative definite. Tensor $G(\mathbf{z})=$

\footnotetext{
${ }^{1}$ Note that it is true for smooth functions $C^{\infty}(X)$, but false for $C^{k}(X)$.
} 
$\sum_{i=1}^{k} \frac{\partial}{\partial z_{i}} \otimes \frac{\partial}{\partial z_{i}}$ for $k<n$, and tensor $G(\mathbf{z})=z_{1}^{2} \frac{\partial}{\partial z_{1}} \otimes \frac{\partial}{\partial z_{1}}+\left(z_{2}^{2} z_{3}^{2}\right) \frac{\partial}{\partial z_{2}} \otimes \frac{\partial}{\partial z_{2}}+$ $\sum_{i=3}^{n} z_{i}^{2} \frac{\partial}{\partial z_{i}} \otimes \frac{\partial}{\partial z_{i}}$ are non-negative definite, but degenerate.

A dissipative vector field generated by the function $h$ is a vector field defined by $X_{h}^{D}(f)=\prec f, h \succ$ for all functions $f$. The flow generated by dissipative vector field should be called a dissipative flow. Dissipative flows essentially differ from the Hamiltonian counterpart: they do not preserve the symmetric structure.

If the $(2,0)$-tensor $G$ is positive-definite, there exists symmetric $(0,2)$-tensor $\mathcal{G}$, its inverse, such that $G(d f, d h)=\mathcal{G}\left(X_{f}^{D}, X_{h}^{D}\right)$, which is exactly a Riemannian metric tensor. In the local coordinates $\left(z_{k}\right)$, if: $G(\mathbf{z})=\sum_{i, j} G^{i j}(\mathbf{z}) \frac{\partial}{\partial z_{i}} \otimes$ $\frac{\partial}{\partial z_{j}}$, the tensor $\mathcal{G}(\mathbf{z})=\sum_{i, j} G_{i j}(\mathbf{z}) d z_{i} \otimes d z_{j}$ where $\sum_{j} G^{i j} G_{j k}=\delta_{k}^{i}$.

The concept of semimetric manifold then is a natural generalization concept of Riemann manifold. It is analogous to a generalization from symplectic manifold to the Poisson manifold.

Similarly as in the Poissonian case, it is not always possible to define induced semimetric structure on a submanifold, hence there is no obvious way to describe canonically constrained semimetric dynamics.

The map $F: M_{1} \rightarrow M_{2}$ between two semimetric manifolds is called semimetric mapping iff it maps the semimetric structures, i.e. $\prec f \circ F, g \circ$ $F \succ_{1}=\prec f, g \succ_{2} \circ F$.

Proposition 3.1 Let $(\mathcal{F}, \cdot, \prec \cdot, \cdot \succ)$ be semimetric algebra.

a) The Schwartz inequality holds

$$
\forall f, g \in \mathcal{F}: \quad \prec f, f \succ \prec g, g \succ \geq \prec f, g \succ^{2} .
$$

b) Let $f_{1}, f_{2}, \ldots, f_{n}$ be arbitrary elements of $\mathcal{F}$. Then the square matrix

$$
\operatorname{Gram}\left(f_{1}, \ldots, f_{n}\right)=\left[\begin{array}{ccc}
\prec f_{1}, f_{1} \succ & \ldots & \prec f_{1}, f_{n} \succ \\
\prec f_{2}, f_{1} \succ & \ldots & \prec f_{2}, f_{n} \succ \\
\ldots & \ldots & \ldots \\
\prec f_{n}, f_{1} \succ & \ldots & \prec f_{n}, f_{n} \succ
\end{array}\right]
$$

is non-negative definite. In particular, $\operatorname{det} \operatorname{Gram}\left(f_{1}, \ldots, f_{n}\right) \geq 0$. Furthermore, if $\mathcal{F}$ is a metric algebra, then $\operatorname{det} \operatorname{Gram}\left(f_{1}, \ldots, f_{n}\right)=0$ iff $\left\{f_{i}\right\}_{i=1}^{n}$ are affine linear dependent. 
Proof. a) Indeed, for each real number $\lambda \in \mathbf{R}$ the expression $0 \leq \prec$ $f-\lambda g, f-\lambda g \succ=\lambda^{2} \prec g, g \succ-2 \lambda \prec f, g \succ+\prec f, f \succ$ is nonnegative quadratic form in the real number $\lambda$. Hence the discriminant $\triangle=4 \prec f, g \succ^{2}-4 \prec f, f \succ \prec g, g \succ \leq 0$ must be non-negative.

If $\mathcal{F}$ is metric algebra, then $\prec f, g \succ^{2}=\prec f, f \succ \prec g, g \succ$ iff $f, g$ are affine linear dependent, i.e. $f-\lambda g=$ Const.

b) For each vector $\mathbf{a}=\left(a_{1}, a_{2}, \ldots, a_{n}\right) \in \mathbf{R}^{n}$, denote $\mathbf{a} \cdot \mathbf{f}=\sum_{i} a_{i} f_{i}$, we have $\mathbf{a}^{T} \operatorname{Gram}\left(f_{1}, \ldots, f_{n}\right) \mathbf{a}=\prec \mathbf{a} \cdot \mathbf{f}, \mathbf{a} \cdot \mathbf{f} \succ \geq 0$.

Q.E.D.

\section{Example 3.2}

1. The natural Euclidean metric of $n$-dim Euclidean space $X=\mathbf{R}^{n}$ induces a natural metric structure on $C^{\infty}(X)$

$$
\prec f, g \succ(\mathbf{x})=\sum_{i=1}^{n} \frac{\partial f}{\partial x_{i}} \frac{\partial g}{\partial x_{i}}, \text { where } f, g \in C^{\infty}\left(\mathbf{R}^{n}\right), \mathbf{x} \in \mathbf{R}^{n}
$$

We shall call the metric bracket (3.4) the Euclidean metric bracket.

2. Here is a simple but general construction of the semimetric structure in the space of smooth functionals over a Hilbert space. Let $X$ be a Hilbert space with a scalar product $\langle\cdot \mid \cdot\rangle$ and $A$ be a linear operator on $X$. One can define a semimetric structure on the space of all smooth functionals over $X$ as following

$$
\prec \Phi, \Psi \succ(\mathbf{f})=\left\langle A \frac{\delta \Phi}{\delta \mathbf{f}} \mid A \frac{\delta \Psi}{\delta \mathbf{f}}\right\rangle .
$$

For instance, let $X$ be a Hilbert space of functions $X=\left\{\mathbf{f}: \mathbf{R}^{n} \rightarrow \mathbf{R}^{d}\right\}$ with a scalar product

$$
\begin{gathered}
(\mathbf{f} \mid \mathbf{g})=\sum_{i, j=1}^{d} \int d^{n} \mathbf{x} f_{i}(\mathbf{x}) G^{i j}(\mathbf{x}) g_{j}(\mathbf{x}), \text { where } \\
\mathbf{f}=\left(f_{1}, f_{2}, \ldots, f_{d}\right), \mathbf{g}=\left(g_{1}, g_{2}, \ldots, g_{d}\right) \in X .
\end{gathered}
$$


The above scalar product on $X$ defines a semimetric bracket on the space of smooth functionals over $X$ by

$$
\prec \Phi, \Psi \succ(\mathbf{f})=\sum_{i, j=1}^{d} \int d^{n} \mathbf{x} \frac{\delta \Phi}{\delta f_{i}(\mathbf{x})} G^{i j}(\mathbf{x}) \frac{\delta \Psi}{\delta f_{j}(\mathbf{x})},
$$

where $\Phi, \Psi \in C^{\infty}(X)$.

To be specific let $d=n$ and $G$ be a differential operator of the form $D^{+} D$, for instance, let $G^{i j}(\mathbf{x})=-\left[a \frac{\partial^{2}}{\partial x_{i} \partial x_{j}}+b \delta_{i j} \triangle\right]$, where $a, b \geq 0$ where $\triangle$ denotes the Laplace operator. The symmetric bracket (3.7) assume now the form

$$
\begin{aligned}
\prec \Phi, \Psi \succ(\mathbf{f})=\int & d^{n} \mathbf{x}\left\{a\left[\nabla \cdot \frac{\delta \Phi}{\delta \mathbf{f}(\mathbf{x})}\right]\left[\nabla \cdot \frac{\delta \Psi}{\delta \mathbf{f}(\mathbf{x})}\right]\right. \\
+b \sum_{i} & {\left.\left[\nabla \frac{\delta \Phi}{\delta f_{i}(\mathbf{x})}\right] \cdot\left[\nabla \frac{\delta \Psi}{\delta f_{i}(\mathbf{x})}\right]\right\} }
\end{aligned}
$$

As we shall see this is exactly a semimetric bracket need in viscous fluid dynamics.

We shall call semimetric dynamics a dynamics which is governed by some semimetric (dissipative) vector field. In local coordinate system $\left(z_{k}\right)$, we have the following system of first-order differential equations:

$$
\dot{z}^{i}=\prec z^{i}, \mathcal{S} \succ=X_{\mathcal{S}}^{D}\left(z^{i}\right)=\sum_{j=1}^{N} G^{i j}(z) \frac{\partial \mathcal{S}}{\partial z^{j}}, i, j=1,2, \ldots, N,
$$

where $\prec \cdot, \cdot \succ$ is semimetric bracket, $X_{\mathcal{S}}^{D}$ is a dissipative vector field generated by function $\mathcal{S}$ defined on the phase space, and $G$ is a semimetric tensor. In some very special cases the function $\mathcal{S}$ has a physical interpretation as entropy. $\mathcal{S}$ is always non-decreasing, since

$$
\dot{\mathcal{S}}=\prec \mathcal{S}, \mathcal{S} \succ \geq 0 .
$$

If $f, g$ are Constants for semimetric dynamics, then the Leibniz rule ensures that $f g$ is also a constant, but $\prec f, g \succ$ is usually not a constant due to the lack of the Jacobi identity. We shall now introduce a new concept a symmetric analogy of the Jacobi Identity which we call the SJ-identity. 


$$
\begin{aligned}
& \text { SJ-identity: } \\
& \forall f, g, h \in \mathcal{F}: 2 \prec \prec f, g \succ, h \succ=[\prec \prec f, h \succ, g \succ+\prec \prec g, h \succ, f \succ] \\
& \Longleftrightarrow \forall f, h \in \mathcal{F}: \quad \prec \prec f, f \succ, h \succ=\prec \prec f, h \succ, f \succ \text {. }
\end{aligned}
$$

A symmetric dynamical system is called a SP-dynamics ("symmetric Poisson dynamics") iff the symmetric Jacobi identity (3.11) holds.

Proposition 3.2 If $f, g$ are Constants of the SP-dynamics, then $\prec f, g \succ$ also is a Constant of motion. In particular, if $f$ is a constant, then $\prec f, f \succ$ also.

Proof. Indeed, since $\prec f, \mathcal{S} \succ=\prec g, \mathcal{S} \succ=0$ we have

$$
\frac{d}{d t} \prec f, g \succ=\prec \prec f, g \succ, \mathcal{S} \succ=\frac{1}{2}[\prec \prec f, \mathcal{S} \succ, g \succ+\prec \prec g, \mathcal{S} \succ, f \succ]=\emptyset 3
$$

\section{Q.E.D.}

Therefore constants of SP-dynamics form a subalgebra. This property is quite useful in finding constants of motion for SP-dynamics.

Note that each autonomous dynamical system described by the system of the first order differential equations

$$
\dot{x}_{k}=F_{k}\left(x_{1}, \ldots, x_{n}\right), k=1, \ldots, n,
$$

is a pseudo-metric system. Indeed, one can choose as the function $\mathcal{S}$, $\mathcal{S}=\sum_{k=1}^{n} x_{k}$, and as a diagonal pseudo-metric $G^{i j}(x)=\delta^{i j} F_{i}(x)$.

Furthermore, locally and almost everywhere each dynamical system (3.13) is metric. Indeed, for each point from the set $\left\{\mathbf{x}: \forall k=1, \ldots, n: F_{k}(\mathbf{x}) \neq 0\right\}$ there exists a neighborhood $U$ such that functions $F_{k}$ do not change their sign inside $U$. Let denote $s_{k}=\operatorname{sign} F_{k}= \pm 1$ in $U$. In the neighborhood $U$, the system should be regarded as metric system with, for instance, $G^{i j}(x)=s_{i} \delta^{i j} F_{i}(x) \geq 0$ and $\mathcal{S}=\sum_{k} s_{k} x_{k}$. In particular, locally and almost everywhere, Poisson dynamics also admit a metric description. Each dynamical system of the type $\dot{x}_{k}=F_{k}(\mathbf{x})$ should be regarded as Poissonian system after doubling the number of variables. Indeed, consider a canonical Poisson structure: $\left\{x_{i}, x_{j}\right\}=0=\left\{p_{i}, p_{j}\right\},\left\{x_{i}, p_{j}\right\}=\delta_{i j}$ and let $\mathcal{H}(\mathbf{x}, \mathbf{p})=\sum_{k} p_{k} F_{k}(\mathbf{x})$, the canonical equations follow

$$
\dot{x}_{k}=\left\{x_{k}, \mathcal{H}\right\}=F_{k}(\mathbf{x}), \dot{p}_{k}=\left\{p_{k}, \mathcal{H}\right\}=-\sum_{j} p_{j} \frac{\partial F_{j}(\mathbf{x})}{\partial x_{k}} .
$$


A system is non-Poissonian (or non-metric) if it can not be written in the Poisson (resp. metric) form without changing the number of variables. Each dynamical system of the type $\dot{x}_{k}=F_{k}(\mathbf{x})$ where functions $F_{k}(\mathbf{x})$ are positivedefinite, is a metric system but it is (in the general) non-Poissonian. The answer to the question which Poissonian system admits a global metric description, remains unknown.

Morrison [9] has pointed out that metric dynamical systems admit an asymptotic stability at isolated maxima of function $\mathcal{S}$. To show that let $\mathbf{x}$ be an isolated maximum of $\mathcal{S}$, then certainly $\partial_{i} \mathcal{S}=0$ at $\mathbf{x}$, hence $\mathbf{x}$ is an equilibrium point of the semimetric dynamical system: $\dot{z}^{i}=\prec z^{i}, \mathcal{S} \succ=\sum_{j} G^{i j}(\mathbf{z}) \partial_{j} \mathcal{S}$. Define the function $L(\mathbf{z})=\mathcal{S}(\mathbf{z})-\mathcal{S}(\mathbf{x})$, then obviously $L(\mathbf{x})=0$ and $L(\mathbf{z})<0$ in some neighborhood of $\mathbf{x}$, since $\mathbf{x}$ is the isolated maximum. Furthermore, $\dot{L}(\mathbf{z})=\prec L, \mathcal{S} \succ(\mathbf{z})=\prec \mathcal{S}, \mathcal{S} \succ(\mathbf{z}) \geq 0$ for $\mathbf{z} \neq \mathbf{x}$ and $\dot{L}(\mathbf{x})=0$, therefore $L$ is Liapunov function for the system and $\mathbf{x}$ is its stable equilibrium point. Now if the system is metric, then $\dot{L}(\mathbf{z})=\prec \mathcal{S}, \mathcal{S} \succ(\mathbf{z})>0$ for $\mathbf{z} \neq \mathbf{x}$ since function $\mathcal{S}$ is not locally constant. Hence $\mathbf{x}$ is asymptotically stable point. Generalization of the above construction to the infinite dimensional case is not known.

\section{Constrained metric dynamics}

In the frame work of symplectic geometry, constrained Hamiltonian dynamics can be represented by a triplet $(M, N, \omega)$ where $(M, \omega)$ is a symplectic manifold, namely Phase space, and $N$ is a constraint submanifold of $M$. Antisymmetric Dirac bracket for second-class constraints [1, 16] is nothing but the Poisson bracket on some symplectic manifold $N^{\prime} \subset N$, called the second-class constraint manifold [16] et. al. (also in [2]).

Similarly, constrained metric dynamics should be represented by a triplet $(M, N, g)$ where $g$ is metric tensor which is responsible for a dissipation and $N$ is a constraint submanifold of $M$. We show that symmetric Dirac bracket for a triple $(M, N, g)$ is nothing but the semimetric bracket on the submanifold $N$. It is worthy to note that any submanifold of a Riemannian manifold is second-class with respect to the metric bracket defined by the metric tensor.

Suppose that we have a pair $(M, \xi)$ where $M$ is a smooth manifold and $\xi$ is non-degenerate symmetric $(0,2)$-tensor on $M$. Then at each point $x \in M$ the map $\xi_{x}: T_{x} M \times T_{x} M \rightarrow R$ is bilinear, symmetric and non-degenerate and it induces a linear bijection $\xi_{x}^{\sharp}: T_{x}^{*} M \rightarrow T_{x} M$. Further, if $\xi$ is Riemann metric 
tensor, then $\xi_{x}$ is a scalar product on $T_{x} M$. The non-degeneracy of the tensor $\xi$ guarantees an existence of a (2,0)-tensor field $\Lambda: T^{*} M \times T^{*} M \rightarrow R$. Let $N$ be a submanifold of $M$ and $\xi_{\mid N}$ is supposed to be non-degenerate. Then at each point $x$ of the submanifold $N$, the linear space $T_{x} M$ decomposes into direct sum of a tangent space to $N, T_{x} N$, and its orthogonal with respect to bilinear symmetric functional $\xi_{x}$, i.e. $T_{x} M=T_{x} N \oplus\left(T_{x} N\right)^{\perp}$. The symmetric Dirac bracket with respect to a triple $(M, N, \xi)$ is defined by

$$
\begin{aligned}
\Lambda_{D}(\alpha, \beta)= & \xi\left(P \xi^{\sharp}(\alpha), P \xi^{\sharp}(\beta)\right), \quad \text { where } P \text { is a projection } \\
& \text { onto } T_{x} N \text { along }\left(T_{x} N\right)^{\perp}, \alpha, \beta \text { are 1-forms on } M .
\end{aligned}
$$

The symmetric Dirac bracket in the space of functions is then

$$
\prec f, g \succ_{D}=\Lambda_{D}(d f, d g), \forall f, g \in C^{\infty}(M) .
$$

If $N$ is the second-class submanifold

$$
N=\left\{x \in M: \Theta_{i}(x)=0\right\},
$$

then we derive explicite formula for symmetric Dirac bracket with respect to the triple $(M, N, \xi)$. Let us denote $X_{g}$ a vector field generated by the function $g$, i.e. $X_{g}(f)=\Lambda(d f, d g)=\prec f, g \succ=\xi\left(X_{f}, X_{g}\right)$. Let $W=\left[W_{i j}\right]=$ $\left[\prec \Theta_{i}, \Theta_{j} \succ\right]=\left[\xi\left(X_{\Theta_{i}}, X_{\Theta_{j}}\right)\right]$ and $C=\left[C_{i j}\right]=W^{-1}$. It is easy to see that the vector fields $X_{\Theta_{i}}$ span $T N^{\perp}$, then orthogonal projection $Q$ onto $T N^{\perp}$ along $T N$ has a form:

$$
Q X=\sum_{i, j} \xi\left(X, X_{\Theta_{i}}\right) C_{i j} X_{\Theta_{j}}
$$

Then the orthogonal projection $P$ onto $T N$ along $T N^{\perp}$ is of the form $P X=$ $X-Q X$, hence we have $P X_{f}=X_{f}-\sum_{i, j} \xi\left(X, X_{\Theta_{i}}\right) C_{i j} X_{\Theta_{j}}$. Then the symmetric Dirac formula is of the form

$$
\begin{aligned}
\prec f, g \succ_{D} & =\Lambda_{D}(d f, d g)=\xi\left(P X_{f}, P X_{g}\right)=\xi\left(X_{f}, P X_{g}\right) \\
& =\xi\left(X_{f}, X_{g}\right)-\xi\left(X_{f}, Q X_{g}\right) \\
& =\prec f, g \succ-\sum_{i, j} \prec f, \Theta_{i} \succ C_{i j} \prec \Theta_{j}, g \succ,
\end{aligned}
$$

which coincides with the antisymmetric Dirac bracket formula (2.4) for Poisson bracket with the antisymmetric brackets $\{\cdot, \cdot\}$ replaced by $\prec \cdot, \cdot \succ$. Our 
procedure shown above applies to both cases, for symmetric or antisymmetric $(0,2)$-tensors.

The Dirac formula (4.5) plays a key role in the practical use of the Dirac brackets. Algebraically, one may use it as a definition of the Dirac bracket for an arbitrary symmetric or antisymmetric algebra. The disadvantage of the algebraic approach is based on the fact that it is very difficult to understand why the Jacobi identity for the new Dirac bracket holds when the above procedure is applied to a Poisson bracket. In the metric context, if the algebraic formula (4.5) is regarded as a definition of the Dirac bracket, then it is easy to check that new algebraic Dirac bracket is symmetric Leibniz bracket (i.e. the algebraic properties i), ii) hold), but the crucial non-negativity property iii) is not easy to verify. A simple proof will be given latter after the Theorem 4.1. It is easy to see that

$$
\prec \Theta_{a}, f \succ_{D}=0 \text {, for arbitrary function } f(z),
$$

therefore all constraints $\Theta_{a}$ are Casimirs, i.e. belong to the Centrum of the semimetric algebra $\left(C^{\infty}(X), \prec \cdot, \cdot \succ_{D}\right)$.

Now we would like to present the new formula for calculating symmetric or antisymmetric Dirac bracket.

\section{Theorem 4.1 [14]}

The following identity holds:

$$
\prec f, g \succ_{D}=\frac{\operatorname{det} W_{f, g}}{\operatorname{det} W}, \forall f, g \in \mathcal{F},
$$

where

$$
\begin{gathered}
W=\left[\begin{array}{cccc}
\prec \Theta_{1}, \Theta_{1} \succ & \ldots & \prec \Theta_{1}, \Theta_{N} \succ \\
\prec \Theta_{2}, \Theta_{1} \succ & \ldots & \prec \Theta_{2}, \Theta_{N} \succ \\
\ldots & \ldots & \ldots \\
\prec \Theta_{N}, \Theta_{1} \succ & \ldots & \prec \Theta_{N}, \Theta_{N} \succ
\end{array}\right], \\
W_{f, g}=\left[\begin{array}{cccc}
\prec \Theta_{1}, \Theta_{1} \succ & \ldots & \prec \Theta_{1}, \Theta_{N} \succ & \prec \Theta_{1}, g \succ \\
\prec \Theta_{2}, \Theta_{1} \succ & \ldots & \prec \Theta_{2}, \Theta_{N} \succ & \prec \Theta_{2}, g \succ \\
\ldots & \ldots & \ldots & \ldots \\
\prec \Theta_{N}, \Theta_{1} \succ & \ldots & \prec \Theta_{N}, \Theta_{N} \succ & \prec \Theta_{N}, g \succ \\
\prec f, \Theta_{1} \succ & \ldots & \prec f, \Theta_{N} \succ & \prec f, g \succ
\end{array}\right] .
\end{gathered}
$$

The same formula holds for antisymmetric Dirac brackets. 
The proof is straightforward; one applies twice the Laplace recursive formula for the determinant expansion to the last column and row of the matrix $W_{f, g}$.

One consequence of (4.7) and (4.8) for semimetric bracket is that $\forall f$ we have $\prec f, f \succ_{D}=\frac{\operatorname{det} W_{f, f}}{\operatorname{det} W}$, and therefore the inequality $\prec f, f \succ_{D} \geq 0$ holds, according to the Proposition 3.1. In one constraint case, this is equivalent to the Schwartz inequality.

The theorem 4.1 provides a new effective formula for calculating Dirac brackets for both symmetric and antisymmetric case. Usually, the direct attempt to use the formula (4.5) is impractical for a system with rather big number of constraints of second type. This is because it requires quite complicated evaluation of the elements of the inverse matrix $C_{i j}$. Note, however, that when we are not interested in the Dirac brackets but only in resulting equations for constrained dynamics then the evaluation of $C=W^{-1}$ is unnecessary. Applying theorem 4.1 we find immediately equation of the quantity $f$ in constrained symmetric/antisymmetric dynamics:

$$
\dot{f}=\prec f, \mathcal{H} \succ_{D}=\frac{\operatorname{det} W_{f, \mathcal{H}}}{\operatorname{det} W}, \quad \text { or } \quad \dot{f}=\{f, \mathcal{H}\}_{D}=\frac{\operatorname{det} W_{f, \mathcal{H}}}{\operatorname{det} W} .
$$

This formula is particularly convenient for finding constants of motion for constrained - symmetric and antisymmetric - dynamics. Indeed, $f$ is a constant of motion for constrained dynamics iff $\operatorname{det} W_{f, \mathcal{H}}=0$.

Below we show few examples of the constrained symmetric Dirac brackets which are applicable in differential geometry and physics.

Example 4.1 Consider the standard Euclidean metrics $\prec z^{i}, z^{j} \succ=\delta^{i j}=$ $G^{i j}$ and the fixed surface $f(\mathbf{z})=0$ in $\mathbf{R}^{n}$. Here the function $f$ is assumed to be smooth and with zero as it regular value, that is $f^{-1}(0)$ is a close regular $n-1$ dimensional differential submanifold in $\mathbf{R}^{n}$. Using $f(\mathbf{z})=0$ as a constraint we find the Dirac semimetric brackets

$$
\prec z^{i}, z^{j} \succ_{D}=\delta^{i j}-n^{i} n^{j},
$$

where $\mathbf{n}(\mathbf{z})=\frac{\nabla f}{\|\nabla f\|}$ is an unit normal vector to the surface at $\mathbf{z}$. The metric tensor $G_{D}^{i j}(\mathbf{z})=\delta^{i j}-n^{i} n^{j}$ is nothing but the induced metric tensor on this surface.

The following example illustrates how to derive the metric structure for lattice-spins [8], that is a set of classical spins $\vec{S}_{a}$ where $a$ labels the lattice sites 
Example 4.2 We introduce the usual lattice-spin metric brackets as

$$
\prec S_{a}^{i}, S_{b}^{j} \succ=\delta_{a b} \delta^{i j}\left|S_{a}\right|=G_{a b}^{i j}, i, j=1,2,3, a, b=1,2, \ldots, N
$$

and we define $2 N$-dim surface $\mathcal{P}$ by the following system of $N$ constraints

$$
\Theta_{a}(\mathbf{S})=\left|S_{a}\right|^{2}-r_{a}^{2}=\sum_{i=1}^{3}\left(S_{a}^{i}\right)^{2}-r_{a}^{2}=0, a=1,2, \ldots, N .
$$

The Dirac metric brackets for the surface $\mathcal{P}$ are

$$
\prec S_{a}^{i}, S_{b}^{j} \succ_{D}=\delta^{i j}\left|S_{a}\right|\left[\delta_{a b}-\frac{S_{a}^{i} S_{b}^{j}}{S_{a}^{2}}\right] .
$$

The next example illustrates the metric structure for energy-conserving rigid body.

\section{Example 4.3}

One may postulate the metric brackets for a rigid body as

$\prec \omega_{i}, \omega_{j} \succ=\delta_{i j} K(\omega)$, where $K$ is some function of the rigid body

$$
\text { angular frequency } \omega \text {. }
$$

a) Consider energy as constrained surface

$$
\Theta(\omega)=\sum_{k=1}^{3} I_{k} \omega_{k}^{2}-E .
$$

Calculating the Dirac metric brackets one gets

$$
\prec \omega_{i}, \omega_{j} \succ_{\{\Theta\}}=K(\omega)\left[\delta_{i j}-\frac{I_{i} I_{j} \omega_{i} \omega_{j}}{\sum_{k} I_{k}^{2} \omega_{k}^{2}}\right] .
$$

We can consider some particular cases:

a1) One may choose $K=\sum_{k=1}^{3} I_{k}^{2} \omega_{k}^{2}$, where $I_{k}$ are moments of inertia with respect to main axes of the rigid body. Then the Dirac metric brackets are

$$
\prec \omega_{i}, \omega_{j} \succ_{\{\Theta\}}=\delta_{i j}\left[\sum_{k=1}^{3} I_{k}^{2} \omega_{k}^{2}\right]-I_{i} I_{j} \omega_{i} \omega_{j} .
$$

This metric structure coincides with the metric structure postulated by Morrison [9]. 
a2) Since the Poisson structure of rigid body is the same as for classical spins, we may postulate $K=|\omega|$, where $|\omega|=\sqrt{\sum_{k=1}^{3} \omega_{k}^{2}}$. The Dirac metric brackets follow

$$
\prec \omega_{i}, \omega_{j} \succ_{\{\Theta\}}=|\omega|\left[\delta_{i j}-\frac{I_{i} I_{j} \omega_{i} \omega_{j}}{\sum_{k=1}^{3} I_{k}^{2} \omega_{k}^{2}}\right] .
$$

b) Consider Poissonian Casimir as constrained surface

$$
\Theta(\omega)=\sum_{k=1}^{3} \omega_{k}^{2}-\left|\omega_{0}\right|^{2}
$$

The Dirac metric brackets follow

$$
\prec \omega_{i}, \omega_{j} \succ_{\{\Theta\}}=K(\omega)\left[\delta_{i j}-\frac{\omega_{i} \omega_{j}}{|\omega|^{2}}\right] .
$$

For instance, $K(\omega)=|\omega|$, we get

$$
\prec \omega_{i}, \omega_{j} \succ_{\{\Theta\}}=|\omega|\left[\delta_{i j}-\frac{\omega_{i} \omega_{j}}{|\omega|^{2}}\right] .
$$

Next examples show how our formalizm works in the Hilbert spaces.

Example 4.4 Let $P h=L^{2}\left(\mathbf{R}^{n} ; \mathbf{R}^{d}\right)$ be a Hilbert space of real, vector valued square integrable functions with standard scalar product $\langle\cdot \mid \cdot\rangle$, i.e. $<\mathbf{f} \mid \mathbf{g}>=\int d^{n} \mathbf{x} \mathbf{f}(\mathbf{x}) \cdot \mathbf{g}(\mathbf{x})$. where $\mathbf{f}=\left(f_{1}, \ldots, f_{d}\right), \mathbf{g}=\left(g_{1}, \ldots, g_{d}\right)$. Let $\|\mathbf{f}\|^{2}=<\mathbf{f} \mid \mathbf{f}>$. In the space of all smooth functionals over $P h$, according to the construction given in the Example 3.2, the semimetric structure may be defined by

$$
\prec \phi_{1}, \phi_{2} \succ(\mathbf{f})=\sum_{i=1}^{d} \int d^{n} \mathbf{x}\left[\frac{\delta \phi_{1}}{\delta f_{i}(\mathbf{x})} \frac{\delta \phi_{2}}{\delta f_{i}(\mathbf{x})}\right], \phi_{1}, \phi_{2}: P h \rightarrow R,
$$

where $\frac{\delta \phi_{i}}{\delta f_{k}}$ denotes a Gateaux functional derivative.

Consider a surface of infinite dimensional sphere $S^{\infty}$ with radius $r$ as a subspace with one constraint

$$
S^{\infty}=\left\{\mathbf{f} \in P h:\|\mathbf{f}\|^{2}=r^{2}\right\} .
$$


Now we calculate the Dirac metric structure for the sphere $S^{\infty}$. The metric bracket (4.22) can be rewritten, introduce the canonical metric tensor $G$

$$
G^{i j}(\mathbf{x}, \mathbf{y})=\prec f_{i}(\mathbf{x}), f_{j}(\mathbf{y}) \succ=\delta_{i j} \delta(\mathbf{x}-\mathbf{y}),
$$

as

$$
\prec \phi_{1}, \phi_{2} \succ(\mathbf{f})=\int d^{n} \mathbf{x} d^{n} \mathbf{y} \frac{1}{2}\left[\frac{\delta \phi_{1}}{\delta f_{i}(\mathbf{x})} \frac{\delta \phi_{2}}{\delta f_{j}(\mathbf{y})}+\frac{\delta \phi_{1}}{\delta f_{j}(\mathbf{y})} \frac{\delta \phi_{2}}{\delta f_{i}(\mathbf{x})}\right] \prec f_{i}(\mathbf{x}), f_{j}(\mathbf{y}) \succ .
$$

The Dirac semimetric structure on $S^{\infty}$ follows

$$
\prec f_{i}(\mathbf{x}), f_{j}(\mathbf{y}) \succ_{D}=\delta_{i j} \delta(\mathbf{x}-\mathbf{y})-\frac{f_{i}(\mathbf{x}) f_{j}(\mathbf{y})}{\|\mathbf{f}\|^{2}} .
$$

Example 4.5 Let $\Psi=\Psi_{1}+i \Psi_{2}$ and its complex conjugate $\Psi^{*}=\Psi_{1}-i \Psi_{2}$ where $\Psi_{1}, \Psi_{2}$ are real functions integrated by square, i.e. they belong to the Hilbert space $L^{2}$. We define metric structure by

$$
\prec \Psi_{k}(\mathbf{x}), \Psi_{l}(\mathbf{y}) \succ=\frac{1}{2} \delta_{k l} \delta(\mathbf{x}-\mathbf{y}), \quad \text { where } k, l=1,2 .
$$

One can rewrite it to the form

$$
\prec \Psi(\mathbf{x}), \Psi(\mathbf{y}) \succ=\prec \Psi^{*}(\mathbf{x}), \Psi^{*}(\mathbf{y}) \succ=0, \prec \Psi(\mathbf{x}), \Psi^{*}(\mathbf{y}) \succ=\delta(\mathbf{x}-\mathbf{y})(4.28)
$$

which we call by canonical metric bracket for Quantum Mechanics. The Dirac structure on the sphere $\|\Psi\|=$ Const in the Hilbert space follows

$$
\begin{array}{r}
\prec \Psi(\mathbf{x}), \Psi(\mathbf{y}) \succ_{D}=-\frac{\Psi(\mathbf{x}) \Psi(\mathbf{y})}{2\|\Psi\|^{2}}, \prec \Psi^{*}(\mathbf{x}), \Psi^{*}(\mathbf{y}) \succ_{D}=-\frac{\Psi^{*}(\mathbf{x}) \Psi^{*}(\mathbf{y})}{2\|\Psi\|^{2}} \\
\prec \Psi(\mathbf{x}), \Psi^{*}(\mathbf{y}) \succ_{D}=\delta(\mathbf{x}-\mathbf{y})-\frac{\Psi(\mathbf{x}) \Psi^{*}(\mathbf{y})}{2\|\Psi\|^{2}}(4.29)
\end{array}
$$

The Dirac brackets of the components $\Psi_{k}$ one get in the accordance with (4.26)

$$
\prec \Psi_{k}(\mathbf{x}), \Psi_{l}(\mathbf{y}) \succ_{D}=\frac{1}{2}\left[\delta_{k l} \delta(\mathbf{x}-\mathbf{y})-\frac{\Psi_{k}(\mathbf{x}) \Psi_{l}(\mathbf{y})}{\|\Psi\|^{2}}\right] .
$$


Example 4.6 (The canonical description for incompressible, viscous fluid dynamics is based on this example)

Let $P h=W^{(1,2)}\left(\mathbf{R}^{n} ; \mathbf{R}^{n}\right)$ be a Sobolev space of real functions. In the space of all smooth functionals over $P h$ we introduce a semimetric structure

$$
\prec \phi_{1}, \phi_{2} \succ(\mathbf{J})=\int d^{n} \mathbf{x}\left\{a\left[\nabla \cdot \frac{\delta \phi_{1}}{\delta \mathbf{J}(\mathbf{x})}\right]\left[\nabla \cdot \frac{\delta \phi_{2}}{\delta \mathbf{J}(\mathbf{x})}\right]+b \sum_{i} \nabla\left[\frac{\delta \phi_{1}}{\delta J_{i}(\mathbf{x})}\right] \cdot \nabla\left[\frac{\delta \phi_{2}}{\delta J_{i}(\mathbf{x})}\right]\right\},
$$

where $\mathbf{J}$ denotes the real vector in the n-dimensional Euclidean space, and $a, b$ are real non-negative coefficients. We can rewrite this semimetric structure in the form

$$
\prec J_{i}(\mathbf{x}), J_{j}(\mathbf{y}) \succ=-\left[a \frac{\partial}{\partial x_{i}} \frac{\partial}{\partial x_{j}}+b \delta^{i j} \triangle\right] \delta(\mathbf{x}-\mathbf{y}) .
$$

Consider infinite-dimensional subspace $\mathcal{V}$ of divergence-free functions (incompressibility condition) as a system with infinite number of constraints:

$$
\mathcal{V}=\left\{\mathbf{J} \in P h \quad: \forall \mathbf{x} \quad \Theta_{\mathbf{x}}(\mathbf{J})=\nabla_{\mathbf{x}} \cdot \mathbf{J}(\mathbf{x})=0\right\} .
$$

Then the Dirac semimetric structure for the subspace $\mathcal{V}$ follows:

$$
\begin{aligned}
\prec J_{i}(\mathbf{x}), J_{j}(\mathbf{y}) \succ_{D}= & -\left[a \frac{\partial}{\partial x_{i}} \frac{\partial}{\partial x_{j}}+b \delta^{i j} \triangle\right] \delta(\mathbf{x}-\mathbf{y})- \\
& \int d \mathbf{z} d \mathbf{z}^{\prime} \prec J_{i}(\mathbf{x}), \Theta(\mathbf{z}) \succ C\left(z, z^{\prime}\right) \prec \Theta\left(\mathbf{z}^{\prime}\right), J_{j}(\mathbf{y}) \succ,
\end{aligned}
$$

where $C$ is an inverse symmetric operator of the constraint matrix

$$
C(\mathbf{x}, \mathbf{y})=\frac{1}{a+b} \int d \mathbf{z} G(|\mathbf{x}-\mathbf{z}|) G(|\mathbf{z}-\mathbf{y}|)
$$

here $G(|\mathbf{x}-\mathbf{y}|)$ denotes the standard Green function (fundamental distribution) of the Laplace equation, i.e. $\triangle_{\mathbf{x}} G(|\mathbf{x}-\mathbf{y}|)=\delta(\mathbf{x}-\mathbf{y})$. Putting eq. (4.35) back to eq. (4.34) and after some simple calculations finally we obtain

$$
\prec J_{i}(\mathbf{x}), J_{j}(\mathbf{y}) \succ_{D}=-b\left[\delta_{i j} \triangle_{\mathbf{x}}-\frac{\partial^{2}}{\partial x_{i} \partial x_{j}}\right] \delta(\mathbf{x}-\mathbf{y})
$$


Physically, the last eq. (4.36) fully describes dissipative structure for incompressible viscous fluid. We shall see that more clearly in subsection 6.5.

\section{Semimetric-Poissonian systems}

The physical systems are usually dissipative. It turns out that both Poissonian and metric structures alone are not enough to describe dissipative systems. However, a proper combination of these two types of dynamics can, for many interesting cases, provide a satisfactory and fully algebraic description of dissipative dynamics.

A semimetric-Poissonian bracket on the space of functions $\mathcal{F}=C^{\infty}(X)$ is a bilinear operation $\{\{\cdot, \cdot\}\}: \mathcal{F} \times \mathcal{F} \rightarrow \mathcal{F}$ which is a linear combination of a Poisson and a semimetric bracket

$$
\forall f, g \in \mathcal{F}:\{\{f, g\}\}=\{f, g\}-\prec f, g \succ,
$$

where $\{\cdot, \cdot\}$ is the Poisson bracket and $\prec \cdot, \cdot \succ$ is the semimetric bracket.

Definition 5.1 Semimetric-Poisson manifold is a pair $(M, \Pi-G)$ where $\Pi$ is a Poisson tensor, $G$ is a semimetric tensor.

We shall call a Semimetric-Poissonian dynamics a dynamics governed by the following system of equations:

$$
\dot{z}^{i}=\left\{\left\{z^{i}, \Phi\right\}\right\}=\left\{z^{i}, \Phi\right\}-\prec z^{i}, \Phi \succ=X_{\Phi}\left(z^{i}\right)-X_{\Phi}^{D}\left(z^{i}\right),
$$

where $\Phi$ is some phase space function. In real physical applications it is often the case that $\Phi$ has the interpretation of the system free energy. It is then a matter of convention to chose minus sign in the eq.(5.2). Indeed we have then

$$
\dot{\Phi}=\{\Phi, \Phi\}-\prec \Phi, \Phi \succ=-\prec \Phi, \Phi \succ \leq 0 .
$$

what describes the dissipation of energy.

It is often even more convenient to go a step further and decompose function $\Phi$ into two parts: the internal energy $\mathcal{H}$ and the dissipation function $\mathcal{S}$, so $\Phi=\mathcal{H}-\mathcal{S}$. Hence, when $\mathcal{S}$ is a Casimir of the Poissonian part, $\{f, \mathcal{S}\}=0$ for all $f$, the evolution of some "observable" $f$ in the semimetric-Poissonian dynamics follows

$$
\dot{f}=\{\{f, \Phi\}\}=\{f, \mathcal{H}\}-\prec f, \mathcal{H}-\mathcal{S} \succ .
$$


Equation (5.4) for $f=\mathcal{S}$ give us the semimetric-Poissonian formulation for the "second law of thermodynamics", namely

$$
\dot{\mathcal{S}}+\prec \mathcal{S}, \mathcal{H} \succ=\prec \mathcal{S}, \mathcal{S} \succ \geq 0
$$

where the expression on the l.h.s. of Eq. (5.5) is just the convective time derivative of $\mathcal{S}$ along the time trajectory in the semimetric-Poissonian phase space.

Note that if $\mathbf{x}$ is an isolated minimum of $\Phi$, then the function $L(\mathbf{z})=$ $\Phi(\mathbf{z})-\Phi(\mathbf{x})$ is a Liapunov function for semimetric-Poisson system. Hence, we obtain

Proposition 5.1 If $\mathrm{x}$ is an isolated minimum of the free energy function $\Phi$, then $\mathbf{x}$ is a stable equilibrium point for semimetric-Poissonian system $\dot{\mathbf{z}}=\{\mathbf{z}, \Phi\}-\prec \mathbf{z}, \Phi \succ$. Furthermore, if the system is metric-Poissonian, then $\mathbf{x}$ is asymptotically stable point.

Example 5.1 Consider a modification of the harmonic oscillator described by

$$
\dot{x}_{1}=x_{2}-a x_{1}\left(x_{1}^{2}+x_{2}^{2}\right), \dot{x}_{2}=-x_{1}-a x_{2}\left(x_{1}^{2}+x_{2}^{2}\right) .
$$

This system is semimetric-Poissonian with

$$
\begin{array}{r}
\left\{x_{1}, x_{2}\right\}=1, \quad \Phi=\frac{1}{2}\left[x_{1}^{2}+x_{2}^{2}\right], \\
\prec x_{1}, x_{1} \succ=a x_{1}^{2}, \prec x_{2}, x_{2} \succ=a x_{2}^{2}, \quad \prec x_{1}, x_{2} \succ=a x_{1} x_{2} .
\end{array}
$$

Point $(0,0)$ is an isolated minimum of $\Phi$, hence it is a stable equilibrium point for the system.

The proposed in preceding sections formal structure of Poissonian and semimetric dynamics allows us to suggest the following scheme for construction of constrained dissipative dynamics of real physical systems.

a) Consider a canonical Poisson structure and semimetric structure. The semimetric structure must be postulated according to our physical insight in the nature of relevant dissipative processes.

b) Choose sets $A, B$ of second-class constraints for the Poissonian and semimetric part, respectively. Note that any constraint is second-class for non-degenerate semimetric structure. 
c) Calculate antisymmetric and symmetric Dirac brackets with respect to the set of constraints $A$ and $B$ and later take a union of them.

Most interesting cases take place when the set of constraints for the semimetric part is a subset of constants of motion for the Poissonian part, i.e. system is dissipative however some of constants of the Poisson dynamics remain constants for the semimetric-Poissonian dynamics. One may use this feature to design many interesting dissipative systems. As an example, we illustrate how to design variety of damped rigid body dynamics in the next section.

\section{Applications: Physical Examples}

\subsection{Particle on the hypersurface with friction}

We shall begin our illustration of the Dirac bracket applications by discussion of a simple example, namely the classical particle moving with friction on a hypersurface $\mathcal{S}_{\text {config }}=\left\{\mathbf{x} \in \mathbf{R}^{n} \mid f(\mathbf{x})=0\right\}$. Denote the particle position by $\mathbf{x}$ and its conjugate momentum by $\mathbf{p}$ and let the friction force experienced by that particle be proportional to the particle velocity. The phase space $\mathbf{R}^{2 n}$ is now equipped with two structures: canonical Poisson and semimetric structure. Then the Poisson structure follows is described by

$$
\left\{x_{i}, x_{j}\right\}=0=\left\{p_{i}, p_{j}\right\},\left\{x_{i}, p_{j}\right\}=\delta_{i j},
$$

and the semimetric structure is defined by

$$
\prec x_{i}, x_{j} \succ=0=\prec x_{i}, p_{j} \succ, \prec p_{i}, p_{j} \succ=\delta_{i j} \lambda_{i},
$$

where $\lambda_{i}(\mathbf{x})>0$ is the directional and space dependent damping coefficient. The semimetric-Poisson structure is defined by $\{\{\cdot, \cdot\}\}=\{\cdot, \cdot\}-\prec \cdot, \cdot \succ$.

Consider $\mathcal{H}=\frac{\mathbf{p}^{2}}{2 m}+V(\mathbf{x})$, the dissipative dynamics derived from the above structures follows

$$
\dot{x}_{i}=\left\{\left\{x_{i}, \mathcal{H}\right\}\right\}=\frac{p_{i}}{m}, \dot{p}_{i}=\left\{\left\{p_{i}, \mathcal{H}\right\}\right\}=-\lambda_{i}(\mathbf{x}) \frac{p_{i}}{m}-\frac{\partial V}{\partial x_{i}},
$$

which can be rewritten in the Newtonian form $m \ddot{x}_{i}+\lambda_{i}(\mathbf{x}) \dot{x}_{i}-F_{i}(\mathbf{x})=0$, where $\mathbf{F}(\mathbf{x})=-\frac{\partial V}{\partial \mathbf{x}}$ is a potential force. In the particular, when $\lambda_{i}(\mathbf{x})=$ $\lambda(\mathbf{x})$, we have $m \ddot{\mathbf{x}}+\lambda(\mathbf{x}) \dot{\mathbf{x}}-\mathbf{F}(\mathbf{x})=0$.

Next consider a fixed algebraic surface $f(\mathbf{x})=0$ in $\mathbf{R}^{n}$. We make assumptions that $f$ is smooth and zero is its regular value. The second assumption 
ensures that $\mathcal{S}_{\text {config }}=f^{-1}(0)$ is a close regular $n-1$ dimensional differential submanifold in $\mathbf{R}^{n}$. Moreover this assumption guarantees $\nabla f \neq 0$, so we can use the gradient to define the normal vector on $\mathcal{S}_{\text {config. }}$.

The set of constraints consists now of two elements:

$$
\Theta_{1} \equiv f(\mathbf{x})=0, \quad \Theta_{2} \equiv \mathbf{p} \cdot \frac{\partial f}{\partial \mathbf{x}}=0
$$

For the Poissonian dynamics both the above constraints $\Theta_{i}$ are second-class in the Dirac classification.

Denoting the unit normal vector to the surface $f$ at the point $x$ by $\mathbf{n}(x)$, $\mathbf{n}(\mathbf{x})=\frac{1}{\left|\frac{\partial f}{\partial \mathbf{x}}\right|} \frac{\partial f}{\partial \mathbf{x}}$, the antisymmetric Dirac brackets for the Poissonian part of our construction are

$$
\begin{aligned}
\left\{x_{i}, x_{j}\right\}_{D} & =0, \quad\left\{x_{i}, p_{j}\right\}_{D}=\delta_{i j}-\frac{1}{\left|\frac{\partial f}{\partial \mathbf{x}}\right|^{2}} \frac{\partial f}{\partial x_{i}} \frac{\partial f}{\partial x_{j}}=\delta_{i j}-n_{i}(\mathbf{x}) n_{j}(\mathbf{x}) \\
\left\{p_{i}, p_{j}\right\}_{D} & =\frac{1}{\left|\frac{\partial f}{\partial \mathbf{x}}\right|^{2}}\left\{\frac{\partial f}{\partial x_{j}}\left[\mathbf{p} \cdot \frac{\partial}{\partial \mathbf{x}}\right] \frac{\partial f}{\partial x_{i}}-\frac{\partial f}{\partial x_{i}}\left[\mathbf{p} \cdot \frac{\partial}{\partial \mathbf{x}}\right] \frac{\partial f}{\partial x_{j}}\right\} \\
& =n_{j}(\mathbf{x})\left[\mathbf{p} \cdot \frac{\partial}{\partial \mathbf{x}}\right] n_{i}(\mathbf{x})-n_{i}(\mathbf{x})\left[\mathbf{p} \cdot \frac{\partial}{\partial \mathbf{x}}\right] n_{j}(\mathbf{x})
\end{aligned}
$$

For the semimetric dynamics only the second constraint $\Theta_{2}$ is second-class. The symmetric Dirac brackets for the semimetric part

$$
\prec x_{i}, x_{j} \succ_{D}=0=\prec x_{i}, p_{j} \succ_{D}, \prec p_{i}, p_{j} \succ_{D}=\delta_{i j} \lambda_{i}-\frac{\lambda_{i}(\mathbf{x}) \lambda_{j}(\mathbf{x}) \frac{\partial f}{\partial x_{i}} \frac{\partial f}{\partial x_{j}}}{\sum_{k} \lambda_{k}(\mathbf{x})\left|\frac{\partial f}{\partial x_{k}}\right|^{2}}
$$

In particular, when $\lambda_{i}(\mathbf{x})=\lambda(\mathbf{x})$, the symmetric Dirac brackets can be written in the form

$$
\prec x_{i}, x_{j} \succ_{D}=0=\prec x_{i}, p_{j} \succ_{D}, \quad \prec p_{i}, p_{j} \succ_{D}=\lambda(\mathbf{x})\left[\delta_{i j}-n_{i}(\mathbf{x}) n_{j}(\mathbf{x})\right] .
$$

Finally take an union of these two Dirac structures

$$
\begin{aligned}
\left\{\left\{x_{i}, x_{j}\right\}\right\}_{D}= & 0, \quad\left\{\left\{x_{i}, p_{j}\right\}\right\}_{D}=\delta_{i j}-n_{i}(\mathbf{x}) n_{j}(\mathbf{x}) \\
\left\{\left\{p_{i}, p_{j}\right\}\right\}_{D}= & \frac{1}{\left|\frac{\partial f}{\partial \mathbf{x}}\right|^{2}}\left\{\frac{\partial f}{\partial x_{j}}\left[\mathbf{p} \cdot \frac{\partial}{\partial \mathbf{x}}\right] \frac{\partial f}{\partial x_{i}}-\frac{\partial f}{\partial x_{i}}\left[\mathbf{p} \cdot \frac{\partial}{\partial \mathbf{x}}\right] \frac{\partial f}{\partial x_{j}}\right\} \\
& -\lambda_{i}(\mathbf{x})\left[\delta_{i j}-\frac{\lambda_{j}(\mathbf{x}) \frac{\partial f}{\partial x_{i}} \frac{\partial f}{\partial x_{j}}}{\sum_{k} \lambda_{k}(\mathbf{x})\left|\frac{\partial f}{\partial x_{k}}\right|^{2}}\right] .
\end{aligned}
$$


When the Hamiltonian for that system has the form $\mathcal{H}=\frac{\mathbf{p}^{2}}{2 m}+V(\mathbf{x})$, the dissipative Hamilton-Dirac equations of motion follow as

$$
\begin{aligned}
\dot{x}_{i}= & \left\{x_{i}, \mathcal{H}\right\}-\prec x_{i}, \mathcal{H} \succ=\frac{1}{m}\left[p_{i}-(\mathbf{p} \cdot \mathbf{n}) n_{i}\right]=\frac{p_{i}}{m} \\
\dot{p}_{i}= & \left\{p_{i}, \mathcal{H}\right\}-\prec p_{i}, \mathcal{H} \succ=F_{i}-\left[\mathbf{F} \cdot \mathbf{n}+\frac{1}{m} \mathbf{p} \cdot\left[\left(\mathbf{p} \cdot \frac{\partial}{\partial \mathbf{x}}\right) \mathbf{n}\right]\right] n_{i} \\
& -\frac{\lambda_{i}(\mathbf{x})}{m}\left[p_{i}-\frac{\partial f}{\partial x_{i}}\left(\frac{\sum_{j} \lambda_{j}(\mathbf{x}) \frac{\partial f}{\partial x_{j}} p_{j}}{\sum_{k} \lambda_{k}(\mathbf{x})\left|\frac{\partial f}{\partial x_{k}}\right|^{2}}\right)\right] .
\end{aligned}
$$

For isotropic damping, when $\lambda_{i}(\mathbf{x})=\lambda(\mathbf{x})$, we can rewrite these equations of motion in the Newtonian form as

$$
m \ddot{\mathbf{x}}+\lambda(\mathbf{x}) \dot{\mathbf{x}}=\mathbf{F}(\mathbf{x})-\left[\mathbf{F}(\mathbf{x}) \cdot \mathbf{n}(\mathbf{x})+m \dot{\mathbf{x}} \cdot \frac{d}{d t} \mathbf{n}(\mathbf{x})\right] \mathbf{n}(\mathbf{x}) .
$$

\subsection{Variety dynamics of damped rigid body}

The usual Poisson brackets for a rigid body angular velocity vector $\vec{\omega}$, after suitable rescaling, are

$$
\left\{\omega_{i}, \omega_{j}\right\}=\sum_{k} \varepsilon_{i j k} \omega_{k}
$$

Suppose that there is no second-class constraints for the Poisson part of the rigid body dynamics and just one such a constraint for its symmetric part. Assume now that this constraint is such that the system energy (or any Casimir function for the metric part) is constant. The choice of that constraint determines details of the semimetric-Poissonian structure.

Using the canonical metric brackets (4.16) and the constrained energy $\mathcal{H}$ from example 4.3 we obtain Dirac metric bracket for rigid body (4.17). Combining these two structures one finds the metric-Poissonian brackets for rigid body [9]:

$$
\begin{aligned}
\left\{\left\{\omega_{i}, \omega_{j}\right\}\right\} & =\left\{\omega_{i}, \omega_{j}\right\}-\lambda \prec \omega_{i}, \omega_{j} \succ_{D} \\
& =\varepsilon_{i j k} \omega_{k}-\lambda\left[\delta_{i j}\left(\sum_{k=1}^{3} I_{k}^{2} \omega_{k}^{2}\right)-I_{i} I_{j} \omega_{i} \omega_{j}\right] .
\end{aligned}
$$

Consider the system "free energy"

$$
\Phi=\mathcal{H}-\mathcal{S}=\frac{1}{2}\left[\sum_{k=1}^{3} I_{k} \omega_{k}^{2}\right]-\mathcal{S}\left(|\omega|^{2}\right),
$$


The equations of, energy conserving, motion for the damped rigid body are

$$
\begin{aligned}
\dot{\omega}_{1}= & \left\{\left\{\omega_{1}, \Phi\right\}\right\}=\left\{\omega_{1}, \mathcal{H}\right\}+\lambda \prec \omega_{1}, \mathcal{S} \succ_{D} \\
= & \omega_{2} \omega_{3}\left(I_{2}-I_{3}\right)+2 \lambda \mathcal{S}^{\prime} \omega_{1}\left[I_{2}\left(I_{2}-I_{1}\right) \omega_{2}^{2}+I_{3}\left(I_{3}-I_{1}\right) \omega_{3}^{2}\right] \\
& \text { and its cyclic permutation } .
\end{aligned}
$$

The dissipation of the free energy follows

$$
\begin{aligned}
\dot{\Phi} & =-\dot{\mathcal{S}}=-\lambda \prec \mathcal{S}, \mathcal{S} \succ_{D} \\
& =-\lambda\left[\left|\frac{\partial \mathcal{S}}{\partial \omega}\right|^{2}\left(\sum_{k=1}^{3} I_{k}^{2} \omega_{k}^{2}\right)-\left(I_{i} \omega_{i} \frac{\partial \mathcal{S}}{\partial \omega_{i}}\right)^{2}\right] \leq 0,
\end{aligned}
$$

and there is no dissipation of energy iff $I_{1}=I_{2}=I_{3}$.

Alternatively, we can derive new semimetric-Poissonian bracket by combining metric bracket (4.18) with the standard Poisson bracket for the rigid body

$$
\begin{aligned}
\left\{\left\{\omega_{i}, \omega_{j}\right\}\right\} & =\left\{\omega_{i}, \omega_{j}\right\}-\lambda \prec \omega_{i}, \omega_{j} \succ_{D} \\
& =\varepsilon_{i j k} \omega_{k}-\lambda|\omega|\left[\delta_{i j}-\frac{I_{i} I_{j} \omega_{i} \omega_{j}}{\sum_{k=1}^{3} I_{k}^{2} \omega_{k}^{2}}\right] .
\end{aligned}
$$

Equations of motion following from the free energy $(\sqrt{6.13})$ are

$$
\begin{aligned}
\dot{\omega}_{1}= & \left\{\left\{\omega_{1}, \Phi\right\}\right\}=\left\{\omega_{1}, \mathcal{H}\right\}+\lambda \prec \omega_{1}, \mathcal{S} \succ_{D} \\
= & \omega_{2} \omega_{3}\left(I_{2}-I_{3}\right)+2 \lambda \mathcal{S}^{\prime}|\omega| \omega_{1} \frac{I_{2}\left(I_{2}-I_{1}\right) \omega_{2}^{2}+I_{3}\left(I_{3}-I_{1}\right) \omega_{3}^{2}}{\sum_{k=1}^{3} I_{k}^{2} \omega_{k}^{2}} \\
& \quad \text { and its cyclic permutation . } \square
\end{aligned}
$$

Finally we derive new semimetric-Poissonian bracket by combining metric bracket (4.21) with the standard Poisson bracket for the rigid body

$$
\begin{aligned}
\left\{\left\{\omega_{i}, \omega_{j}\right\}\right\} & =\left\{\omega_{i}, \omega_{j}\right\}-\lambda \prec \omega_{i}, \omega_{j} \succ_{D} \\
& =\varepsilon_{i j k} \omega_{k}-\lambda|\omega|\left[\delta_{i j}-\frac{\omega_{i} \omega_{j}}{|\omega|^{2}}\right] .
\end{aligned}
$$

Again using the free energy $(6.13)$ we find

$$
\begin{aligned}
\dot{\omega}_{1}= & \left\{\left\{\omega_{1}, \Phi\right\}\right\}=\left\{\omega_{1}, \mathcal{H}\right\}-\lambda \prec \omega_{1}, \mathcal{H} \succ_{D} \\
= & \omega_{2} \omega_{3}\left(I_{2}-I_{3}\right)-\lambda|\omega| \omega_{1}\left[I_{1}-\frac{\sum_{k=1}^{3} I_{k} \omega_{k}^{2}}{|\omega|^{2}}\right] \\
& \text { and its cyclic permutation } .
\end{aligned}
$$


The above dynamics does not conserve the energy, but $|\omega|^{2}$ remains the system Casimir. The energy dissipation is given by

$$
\dot{\mathcal{H}}=-\lambda \prec \mathcal{H}, \mathcal{H} \succ_{D}=-\lambda|\omega|\left[\sum_{k} I_{k}^{2} \omega_{k}^{2}-\frac{\left(\sum_{k} I_{k} \omega_{k}^{2}\right)^{2}}{|\omega|^{2}}\right] \leq 0,
$$

since $\left(\sum_{k} I_{k}^{2} \omega_{k}^{2}\right)|\omega|^{2} \geq\left(\sum_{k} I_{k} \omega_{k}^{2}\right)^{2}$. For $\lambda>0,|\omega|>0$, there is no dissipation of energy when $I_{1}=I_{2}=I_{3}$.

\subsection{Classical damped spins}

The Poisson bracket for lattice-spins

$$
\left\{S_{a}^{i}, S_{b}^{j}\right\}=\delta_{a b} \varepsilon_{i j k} S_{a}^{k},
$$

where $a$ labels the spin location and $k=1,2,3$, guarantees that length of each spin $\left|\mathbf{S}_{a}\right|$ is a Casimir. Our model is based on the assumption that there are no second-class constraints for the Poissonian part and only one constraint for the metric part.

Starting from canonical metric bracket (4.11) and using the length of the spins $\left|\mathbf{S}_{a}\right|$ constraint, as in the example 4.2, we obtain the Dirac metric bracket for lattice-spins (4.13). Combining these two structures one finds the metric-Poissonian of lattice spins [8]:

$$
\begin{aligned}
\left\{\left\{S_{a}^{i}, S_{b}^{j}\right\}\right\} & =\left\{S_{a}^{i}, S_{b}^{j}\right\}-\lambda \prec S_{a}^{i}, S_{b}^{j} \succ_{D} \\
& =\delta_{a b} \varepsilon_{i j k} S_{a}^{k}-\lambda \delta^{i j}\left|\mathbf{S}_{a}\right|\left[\delta_{a b}-\frac{S_{a}^{i} S_{b}^{j}}{S_{a}^{2}}\right],
\end{aligned}
$$

where $\lambda$ is the damping coefficient.

Now we can easily derive the Landau-Lifshitz-Gilbert equation of classical damped lattice-spins

$$
\dot{\mathbf{S}}_{a}=\left\{\left\{\mathbf{S}_{a}, \mathcal{H}\right\}\right\}=\mathbf{S}_{a} \times \mathbf{B}_{e f, a}-\lambda \frac{\mathbf{S}_{a} \times\left(\mathbf{S}_{a} \times \mathbf{B}_{e f, a}\right)}{\left|\mathbf{S}_{a}\right|}
$$

where $\mathbf{B}_{e f, a}=-\frac{\partial \mathcal{H}}{\partial \mathbf{S}_{a}}$ is the effective magnetic field acting on the spin $\mathbf{S}_{a}$. The dissipation of energy follows

$$
\dot{\mathcal{H}}=-\lambda \prec \mathcal{H}, \mathcal{H} \succ_{D}=-\lambda \sum_{a}\left|S_{a}\right|\left[\mathbf{B}_{e f, a}^{2}-\frac{\left(\mathbf{B}_{e f, a} \cdot \mathbf{S}_{a}\right)^{2}}{\left|\mathbf{S}_{a}\right|^{2}}\right] \leq 0 .
$$




\subsection{Dissipative quantum mechanics}

Combining canonical Poisson brackets for quantum mechanics [QM]

$$
\{\Psi(\mathbf{x}), \Psi(\mathbf{y})\}=\left\{\Psi^{*}(\mathbf{x}), \Psi^{*}(\mathbf{y})\right\}=0,\left\{\Psi(\mathbf{x}), \Psi^{*}(\mathbf{y})\right\}=\frac{1}{i \hbar} \delta(\mathbf{x}-\mathbf{y}),
$$

and constrained metric brackets for QM (4.29), which were derived from the canonical metric bracket for QM (4.28) for the physically important constraint, namely the conservation of the wave function norm (probability conservation) as in the example 4.5, we find dissipative Metric-Poissonian structure for $Q M$ :

$$
\begin{aligned}
\{\{\Psi(\mathbf{x}), \Psi(\mathbf{y})\}\} & =\{\Psi(\mathbf{x}), \Psi(\mathbf{y})\}-\frac{\lambda}{\hbar} \prec \Psi(\mathbf{x}), \Psi(\mathbf{y}) \succ_{D}=\frac{\lambda}{\hbar} \frac{\Psi(\mathbf{x}) \Psi(\mathbf{y})}{2\|\Psi\|^{2}} \\
\left\{\left\{\Psi^{*}(\mathbf{x}), \Psi^{*}(\mathbf{y})\right\}\right\} & =\left\{\Psi^{*}(\mathbf{x}), \Psi^{*}(\mathbf{y})\right\}-\frac{\lambda}{\hbar} \prec \Psi^{*}(\mathbf{x}), \Psi^{*}(\mathbf{y}) \succ_{D}=\frac{\lambda}{\hbar} \frac{\Psi^{*}(\mathbf{x}) \Psi^{*}(\mathbf{y})}{2\|\Psi\|^{2}} \\
\left\{\left\{\Psi(\mathbf{x}), \Psi^{*}(\mathbf{y})\right\}\right\} & =\left\{\Psi(\mathbf{x}), \Psi^{*}(\mathbf{y})\right\}-\frac{\lambda}{\hbar} \prec \Psi(\mathbf{x}), \Psi^{*}(\mathbf{y}) \succ_{D} \\
& =\frac{1}{i \hbar} \delta(\mathbf{x}-\mathbf{y})-\frac{\lambda}{\hbar}\left[\delta(\mathbf{x}-\mathbf{y})-\frac{\Psi(\mathbf{x}) \Psi^{*}(\mathbf{y})}{2\|\Psi\|^{2}}\right]
\end{aligned}
$$

where $\lambda$ is the (undefined) damping constant.

Using the conventional Hamiltonian for the Schrödinger equation

$$
\mathcal{H}\left(\Psi, \Psi^{*}\right)=<\Psi|H| \Psi>=\int d^{n} \mathbf{x} \Psi^{*}(\mathbf{x}) H \Psi(\mathbf{x}),
$$

where $H$ is the quantum mechanical (self-adjoined) operator we obtain the evolution of the wave function in the form:

$$
\left.i \hbar \partial_{t} \Psi(\mathbf{x})=i \hbar\{\{\Psi(\mathbf{x}), \mathcal{H}\}\}=H \Psi(\mathbf{x})+i \lambda\left[\frac{<\Psi|H| \Psi>}{\|\Psi\|^{2}}-H\right] \Psi((66)) 28\right)
$$

This equation is known as the Gisin dissipative wave equation [1]. Here the construction of the semimetric bracket ensures that the norm of the state vector is reserved (so the probability is conserved) since it is a second-class constraint for the semimetric part. The dissipation of energy follows

$$
\dot{\mathcal{H}}=-\prec \mathcal{H}, \mathcal{H} \succ=\frac{2 \lambda}{\hbar}\left[-\|H \Psi\|^{2}+\frac{<\Psi|H| \Psi>^{2}}{\|\Psi\|^{2}}\right] \leq 0,
$$

(Schwartz inequality). 
In the above equation the equality is achieved for $\Psi$ which are the eigenstates of the Hamiltonian. The damping in the Gisin equation refers, therefore, to the transition amplitudes only. When the initial wave packet is constructed from eigenstates corresponding to energies $E \geq E_{0}$ then the final state of the evolution described by eq. 6.28) is the eigenstate with energy $E_{0}$. This property distinguishes the Gisin dissipative wave equation from other dissipative Schrödinger equations.

\subsection{Viscous fluid dynamics}

In the fluid mechanics the state of an isothermal fluid is described by its mass density and velocity fields $(\varrho, \mathbf{u})$ or by $(\varrho, \mathbf{J})$ where the current $\mathbf{J}$ field equals $\mathbf{J}=\varrho \mathbf{u}$. The semimetric structure for fluid dynamics 8 follows

$$
\begin{array}{r}
\prec \varrho(\mathbf{x}), \varrho(\mathbf{y}) \succ=0, \quad \prec \varrho(\mathbf{x}), J_{k}(\mathbf{y}) \succ=0, \\
\prec J_{k}(\mathbf{x}), J_{l}(\mathbf{y}) \succ=-\left[a \frac{\partial^{2}}{\partial x_{k} \partial x_{l}}+\eta \delta_{k l} \triangle_{x}\right] \delta(\mathbf{x}-\mathbf{y}),
\end{array}
$$

where $a=\zeta+\frac{\eta}{3}$ and $\zeta, \eta$ are bulk and shear viscosity respectively.

The structure (6.30) is semimetric, indeed $\forall \mathcal{G}$ we have:

$$
\begin{aligned}
\prec \mathcal{G}, \mathcal{G} \succ(\varrho, \mathbf{J}) & =-\sum_{k, l} \int d \mathbf{x} d \mathbf{y} \frac{\delta \mathcal{G}}{\delta J_{k}(\mathbf{x})}\left\{\left[a \frac{\partial^{2}}{\partial x_{k} \partial x_{l}}+\eta \delta_{k l} \triangle_{x}\right] \delta(\mathbf{x}-\mathbf{y})\right\} \frac{\delta \mathcal{G}}{\delta J_{l}(\mathbf{y})} \\
& =\int d \mathbf{x}\left\{a\left[\nabla \cdot \frac{\delta \mathcal{G}}{\delta \mathbf{J}(\mathbf{x})}\right]^{2}+\eta \sum_{k}\left|\nabla\left[\frac{\delta \mathcal{G}}{\delta J_{k}(\mathbf{x})}\right]\right|^{2}\right\} .
\end{aligned}
$$

Note, that this is not a metric bracket, since any functional which depends only on $\varrho$, has zero semimetric bracket with himself. Also note that the kinetic fluid energy $E_{k i n}=\int d \mathbf{x} \mathbf{J}^{2} / 2 \varrho$ dissipation follows directly from (6.30):

$\dot{E}_{k i n}=-\prec E_{k i n}, E_{k i n} \succ=-\int d \mathbf{x}\left\{a\left[\nabla \cdot \frac{\mathbf{J}}{\varrho(\mathbf{x})}\right]^{2}+\eta \sum_{k}\left|\nabla \frac{J_{k}}{\varrho(\mathbf{x})}\right|^{2}\right\} \leq 0$.

In classical hydrodynamics a particular role is played by the incompressible fluid assumption, for incompressible viscous fluid according to eq. (4.36) we 
have

$$
\begin{array}{r}
\prec \varrho(\mathbf{x}), \varrho(\mathbf{y}) \succ_{D}=0, \quad \prec \varrho(\mathbf{x}), J_{i}(\mathbf{y}) \succ_{D}=0 \\
\prec J_{i}(\mathbf{x}), J_{j}(\mathbf{y}) \succ_{D}=-\eta\left[\delta_{i j} \triangle_{\mathbf{x}}-\frac{\partial^{2}}{\partial x_{i} \partial x_{j}}\right] \delta(\mathbf{x}-\mathbf{y}) .
\end{array}
$$

The dissipative energy for viscous incompressible fluid should be easily calculated

$$
\dot{E}_{k i n}=-\prec E_{k i n}, E_{k i n} \succ_{D}=-\frac{\eta}{2} \int d \mathbf{x}\left\{\sum_{k, l}\left(\frac{\partial u_{k}}{\partial x_{l}}+\frac{\partial u_{l}}{\partial x_{k}}\right)^{2}\right\} \leq 0 .
$$

Finally, put $\nu=\frac{\eta}{\varrho_{0}}$ the kinematic viscosity, using (6.33) and the Poisson part calculated in [7] we easily derive non-local evolutional equations for incompressible viscous fluid

$$
\frac{\partial \mathbf{u}}{\partial t}+(\mathbf{u} \cdot \nabla) \mathbf{u}-\nu \triangle \mathbf{u}=\nabla \int d \mathbf{z} G(\mathbf{x}-\mathbf{z}) \nabla_{\mathbf{z}}\left[(\mathbf{u} \cdot \nabla) \mathbf{u}-\nu \triangle_{\mathbf{z}} \mathbf{u}\right] .
$$

\section{$7 \quad$ Final comments}

The description of the dissipative systems dynamics is usually undertaken within the framework of non-equilibrium statistical mechanics. The "simplified" version of the full many-body description often used in applications, for example in phase transformations physics, statistical theory of turbulence, granular media dynamics etc. is the kinetic equation for the time evolution of the "relevant degrees of freedom" distribution function. This equation is derived making strict assumption about the nature of the fluctuations in the system - the underlying stochastic process performed by the relevant system degrees of freedom in the full phase space of the physical model. The equations of motion for the coarse grained variables describing the meso- or macroscopic system properties are obtained from that kinetic equation by one of the known procedures, which once are well justified and understood, and occasionally are just a heuristic chain of semi-mathematical operations.

In this paper we have discussed a novel approach to description of the dissipative systems dynamics, which is purely algebraic. Instead of making series of assumptions on the level of microscopic physics of the problem we 
assume existence of certain algebraic structure, akin to that used in Hamiltonian formulation of classical dynamics which permit us to derive meso- or macroscopic dissipative equations directly from the system free energy. The basic ingredient of that procedure, the semimetric Leibniz bracket for dynamics variables over the whole phase space, is postulated according to our knowledge about dissipative processes. With knowledge of the Poissonian structure for non-dissipative part of the system dynamics, the symmetries of the problem and using Dirac machinery [1] one can derive easily different Dirac structures, which are otherwise hard to postulated, describing dissipative dynamics. One may use the algorithm proposed here to design variety dissipative dynamical systems with required conservative observables.

As shown in the paper this permits us to build up the metriplectic dynamics scenario for several non-trivial systems: classical particle physics, many spins dynamics, rigid body dynamics, compressible and incompressible viscous fluid dynamics and some quantum mechanical problems. Several other applications, notably the relativistic charged particle systems which can also be formulated within the metriplectic scenario have been discussed previously [17, 18]. The quantum applications are of particular interest in view of some similarity between the metriplectic approach and the Lindblads construction [19] - the standard tool in dissipative quantum system analysis. We expect to comment on the connection between both these approaches in the forthcoming publication.

\section{Appendix}

A symmetric Leibniz bracket which satisfies the SJ-identity is called the $S P$ bracket. The tensor $G$ which generates SJ-bracket is called SP-tensor. In the local coordinates

$$
\forall_{i, j=1,2, \ldots, N}: 0=\sum_{k=1}^{N}\left[G^{k j} \frac{\partial G^{i i}}{\partial z^{k}}-G^{k i} \frac{\partial G^{i j}}{\partial z^{k}}\right] .
$$

Hence, SP-bracket and SP-tensor are symmetric analogy to Poisson bracket and Poisson tensor, respectively. In particular each symmetric tensor where $G_{i j}$ are constants (i.e. do not depends on points), generates a SP-bracket. For a non-trivial example of SJ-tensor, see Example 7.1.

$S P$-manifold is a pair $(M, G)$ where $G$ is a symmetric SP-tensor of the type $(2,0)$. 
Example 7.1 It is easy to see that $G=\sum_{i, j=1}^{N} z_{i} z_{j} \frac{\partial}{\partial z_{i}} \otimes \frac{\partial}{\partial z_{j}}$ is SP-tensor. Hence, $\left(\mathbf{R}^{N}, G\right)$ is SP-manifold.

Similarly, with the concept of SJ-identity one can define SL-algebra, SPalgebra which are symmetric analogy of Lie algebra and Poisson algebra, respectively. To prove that there is no another symmetric Jacobi identity we need few elementary algebraic concepts. By an identity of the algebra $\mathcal{F}$ we mean a polynomial $P$ in some free algebra which is identically zero when the generators are replaced by any elements of $\mathcal{F}$. We are interested on the 3-linear identities in which each term involves two pairs of brackets, i.e. of the form: $a \prec \prec f, g \succ, h \succ+b \prec \prec f, h \succ, g \succ+c \prec \prec g, h \succ, f \succ=0$. Now we would like prove the following result:

Proposition 7.1 There are exactly two types of 3-linear identities for symmetric algebra in which each term involves two pairs of brackets:

a) The SE-identity (symmetric version of the Engel identity): $\forall f \in \mathcal{F}$ : $\prec \prec f, f \succ, f \succ=0$.

b) The SJ-identity (symmetric version of the Jacobi identity):

$$
\begin{array}{r}
\forall f, g: \quad \prec \prec f, f \succ, g \succ=\prec \prec f, g \succ, f \succ \Longleftrightarrow \\
\forall f, g, h: 2 \prec \prec f, g \succ, h \succ=[\prec \prec f, h \succ, g \succ+\prec \prec g, h \succ, f \succ] .
\end{array}
$$

Proof. $\quad$ Suppose that $a \prec \prec f, g \succ, h \succ+b \prec \prec f, h \succ, g \succ+c \prec \prec g, h \succ$ , $f \succ=0$. For $f=g=h$ we have $(a+b+c) \prec \prec f, f \succ, f \succ=0$, therefore the identity must be of the type a) or $a+b+c=0$. If $a+b+c=0$, then for $f=h$ we have $(a+c) \prec \prec f, g \succ, f \succ+b \prec \prec f, f \succ, g \succ=0$, i.e. it must be of the type $\mathbf{b})$, since $a+c=-b$.

\section{Q.E.D.}

Similarly, one can prove that there are exactly two 3-linear identities in which each term involves two pairs of brackets for antisymmetric algebra:

a) The Engel identity:

$$
\forall f, g:\{\{f, g\}, f\}=0 \Longleftrightarrow \forall f, g, h:\{\{f, g\}, h\}+\{\{h, g\}, f\}=0 .
$$

b) The Jacobi identity:

$$
\forall f, g, h:\{\{f, g\}, h\}+\{\{g, h\}, f\}+\{\{h, f\}, g\}=0 .
$$


Even the Jacobi identity and $S J$-identity are formally similar as we have seen above, but there exists also a fundamental difference between them. Indeed, the former is equivalent to $X_{h}\{f, g\}=\left\{X_{h} f, g\right\}+\left\{f, X_{h} g\right\}$, i.e. Hamiltonian vector fields act as derivations for Poisson bracket, but for the latter $2 X_{h}^{D} \prec f, g \succ=\prec X_{h}^{D} f, g \succ+\prec f, X_{h}^{D} g \succ$. In the other words, the Jacobi identity manifests some basic features like cyclicity, derivation and signature property while the $S J$-identity does not.

Furthermore, the most natural symmetric bracket $\prec A, B \succ=\frac{A B+B A}{2}$ does not satisfy the $S J$-identity in the general. Hence, there is no natural representation for $S P$-algebras. 


\section{References}

[1] P A M Dirac 1950 Canad. J. Math 2, 129;

1958 Proc. Roy. Soc. London, ser. A 246, 326;

1964 "Lecture Notes on Quantum Mechanics", Yeshiva Univ., New York.

[2] J E Marsden, T S Ratiu 1994 "Introduction to Mechanics and Symmetries", p 226, Springer, Heidelberg.

[3] K H Bhaskara, K Viswanath 1988 "Poisson Algebras and Poisson Manifolds", Longman Scientific and Technical, London.

[4] E C G Sudarshan, N Mukunda 1974 "Classical Dynamics: a modern perspective", Chapter 9, John Wiley \& Sons, New York.

[5] A A Deriglazov, A V Galajinsky, S L Lyakhovich 1996 Nucl. Phys. B 473, 245-266.

[6] F Ferrari, I Lazziezzera 1997 Phys. Lett. B 395, 250-256.

[7] S Q H Nguyen and E A Turski 1999 Physica A 272, 48-55; 2001 Physica A 290, 431-444.

[8] C P Enz, Ł A Turski 1979 Physica A 96, 369;

Ł A Turski 1990 in: Continuum Models and Discrete Systems, Vol. 1 G A Maugin editor, Longman, London;

Ł A Turski 1996 Springer Lectures in Physics 4777, Z Petru, J Przystawa and K Rapcewicz editors, Springer, New York.

[9] Philip J Morrison 1986 Physica D 18, 410-419.

[10] C P Enz 1977 Physica A 89, 1.

[11] N Gisin 1981 J. Phys. A 14, 2259; 1982 Physica A 111, 364.

[12] J A Hołyst, Ł A Turski 1986 Phys. Rev. B 34, 1937.

[13] S Q H Nguyen and Ł A Turski, in prep.

[14] S Q H Nguyen and E A Turski, in prep.

[15] A Weinstein 1983 J. Diff. Geometry 18, 523-557. 
[16] J Śniatycki 1974 Annales de l'Inst. Henri Poincar, vol. XX, no. 4, 365-372.

[17] I Białynicki-Birula, Ł A Turski, and J C Hubbard 1984 Physica A 128, 509 .

[18] Ł A Turski, A N Kaufman 1987 Phys. Lett. A 120, 331.

[19] G Lindblad 1976 Commun. Math. Phys., vol. 48, 119-130. 